\title{
Complications of 5-Fluorouracil After Trabeculectomy
}

\author{
W. A. FRANKS and R. A. HITCHINGS \\ London
}

\begin{abstract}
Summary
Trabeculectomy has a very high success rate, ${ }^{1-3}$ however, certain eyes are known to be at high risk of failure due to scarring of the conjunctival bleb. Such eyes include those with a previous failed filter, eyes with glaucoma secondary to uveitis and neovascularisation and the eyes of children and young adults. Trabeculectomy is generally accepted to have less successful results in patients of African race although good results are reported from some centres. ${ }^{48}$

The effectiveness of treatment with 5-fluorouracil in improving the results of trabeculectomy has been established in a number of pilot studies and clinical trials. ${ }^{9-16}$ Early studies used $100 \mathrm{mg}$ of 5-fluorouracial in divided doses. Serious complications were recorded and lower dose regimes have been advised to improve the safety of the technique. Similar success in improving the chance of success to trabeculectomy has been found with doses of $40 \mathrm{mg}$ with a lower incidence of side effects. ${ }^{14-16}$

We report complications associated with the use of 5-fluorouracil in a total dose of $50 \mathrm{mg}$ in a group of 49 eyes undergoing trabeculectomy and postoperative 5-fluorouracil and describe the previously unreported increased incidence of thin cystic blebs in these eyes.
\end{abstract}

\section{Patients and methods}

Eyes were selected for treatment with postoperative 5-fluorouracil injections because they were judged to be at high risk of failure of trabeculectomy from scarring of the conjunctival bleb. Forty-nine eyes of 45 patients were treated. The indications for use of 5-fluorouracil are shown in Table I. Thirty eight eyes underwent trabeculectomy alone and 11 eyes was combined with extracapsular lens extraction and posterior chamber lens implant. Mean follow-up was 10 months (range 4-16 months).

Average age was 49 years (range 14-82). Twenty eight males and 21 females were treated. Mean number of preoperative medications was 2.4 and the mean preoperative IOP was $29 \mathrm{mmHg}$.

Forty eight operations were performed under general anaesthesia and one case by subconjunctival infiltration with local anaes-

Table I Indications for use of 5-Fluorouracil in 49 eyes

\begin{tabular}{lr} 
Repeat filtering surgery & 21 \\
Secondary glaucoma due to uveitis & 11 \\
Aphakia & 4 \\
Age $<40$ & 3 \\
African Race & 3 \\
Multiple indications & 7 \\
\hline
\end{tabular}


thetic. A Cairns type trabeculectomy ${ }^{17}$ was performed, using a fornix based conjunctival flap and rectangular limbal placed scleral flap. Both sclera and conjunctiva were closed with $10 / 0$ nylon sutures. The technique of combined trabeculectomy and extracapsular cataract extraction is described elsewhere. ${ }^{18}$

Subconjunctival injections of 5-fluorouracil were commenced on the first postoperative day. Five milligrams were administered from a solution of $25 \mathrm{mg} / \mathrm{ml}(0.2 \mathrm{ml})$ drawn up into an insulin syringe and a subconjunctival injection given into the lower fornix. Injections were continued daily for seven days and then on alternative days on days 9,11 and 13. The total dose administered was $50 \mathrm{mg}$. Injections were discontinued if confluent areas of corneal erosions appeared.

Eyes were examined daily for two weeks, then at one month, three months, six months and twelve months and more frequently if judged clinically necessary. Postoperative topical dexamethasone was continued four times daily for one month and three times daily for a further two months. Chloramphenicol drops were commenced on the day before surgery and continued for two weeks.

Intraocular pressure measurements were by Goldmann applanation tonometry.

Bleb morphology was compared to a control group, retrospectively reviewed from the hospital notes, who were matched for age, type of glaucoma, number of previous operations, surgical technique and successful outcome. The purpose of this was to see if the incidence off cystic drainage blebs in eyes with satisfactory intraocular pressure control was higher in eyes treated with 5-fluorouracil. The preoperative characteristics of the study and control groups are given in Table II.

Table II Preoperative characteristics of 5-fluorouracil treated eyes and control eyes

\begin{tabular}{lcc}
\hline & 5-fluorouracil & Controls \\
\hline Number & 49 & 47 \\
Age & 51.3 & 49.0 \\
No. of previous & & \\
$\quad$ glaucoma operations & 0.53 & 0.59 \\
No. medications & 2.2 & 2.6 \\
Preoperative intraocular & & \\
$\quad$ pressure & 27.4 & 28.0 \\
Primary glaucoma & 29 & 26 \\
Secondary glaucoma & 18 & 23 \\
\hline
\end{tabular}

\section{Results}

An intraocular pressure of less than $21 \mathrm{mmHg}$ with or without additional topical medication was achieved in $90 \%$ at last follow up (mean 10 months-range 4-21 months).

Four eyes required further surgery to control intraocular pressure, one patient required oral acetazolamide as well as topical medication and eight eyes required topical medication alone.

Visual acuity was $6 / 12$ or better in 24 eyes at last examination. One eye had improved visual acuity due to clearance of corneal blood staining after a black ball hyphaema and ten of eleven eyes undergoing combined trabeculectomy and cataract extraction had improved acuity. Two eyes developed further field loss with deterioration of vision. Both had early failure due to fibrous scarring and had subsequent tube implant surgery. Complications of 5-fluorouracial are shown in Table III.

\section{Early complications:}

\section{Corneal erosions:}

Corneal erosions were observed in eight eyes $(16 \%)$. All responded to treatment with pad and bandage and there were no long term sequelae. It was considered necessary to discontinue 5-fluorouracil injections to one eye because of a painful large corneal epithelial deficit.

\section{Early wound leakage:}

Early wound leakage was seen in 12 cases $(25 \%)$. Ten were associated with shallowing of the anterior chamber. One eye required resuturing of the scleral flap to overcome corneal lens touch on the first postoperative day.

Table III Complications of 5-fluorouracil in 49 eyes

5 -

fluorouracil Controls p-value

Early complications:

Corneal erosions

Bleb leakage

Bleb rupture

8

Late:

$\begin{array}{llll}\text { Cystic blebs } & 20 & 9 & 0.03\end{array}$

Dellen formation $\quad 3 \quad 0$

Hypotony

$($ IOP $<9 \mathrm{mmHg})$

10

3 


\section{Bleb rupture:}

Three eyes developed breakdown of the conjunctival epithelium over the drainage bleb with leakage of aqueous humour into the tear film and shallowing of the anterior chamber. This complication occurred at three, six and 12 weeks after surgery.

\section{Late complications:}

\section{Cystic bleb formation: (Fig 1)}

Cystic blebs formed in 20 eyes compared to nine eyes in the control group $(\mathrm{p}<0.05 \mathrm{Chi}$ Square). Mean IOP in eyes with cystic blebs was $11.2 \mathrm{mmHg}$ compared to $14.2 \mathrm{mmHg}$ in those with functioning non cystic blebs. Mean IOP in eyes with cystic blebs in the control group was $12.7 \mathrm{mmHg}$ and $14.5 \mathrm{mmHg}$ in eyes with functioning non-cystic blebs.

\section{Hypotony:}

An intraocular pressure of $9 \mathrm{mmHg}$ or less was found in 10 eyes, all with Seidel positive cystic blebs, three months or more after surgery. Three cases or hypotony with cystic blebs occurred in the control group. ( $p=0.8$ Chi Square)

\section{Dellen formation:}

Three eyes treated with 5-fluorouracil developed Dellen corneal erosions adjacent to large cystic blebs compared to one eye in the control group.

\section{Discussion}

5-fluorouracil is effective in improving the

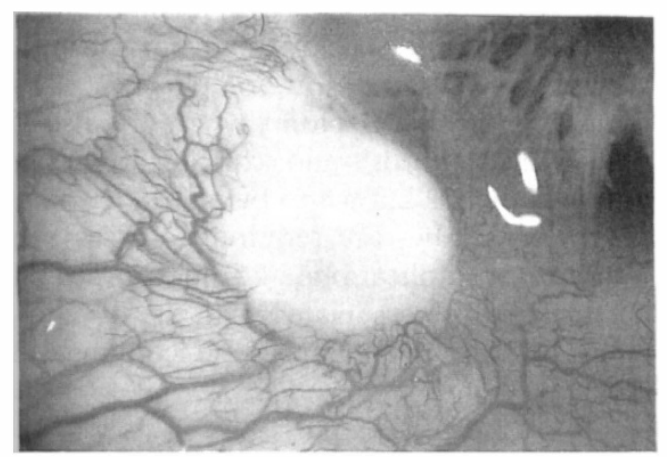

Fig. 1. Cystic bleb showing surrounding scarring and distortion of conjunctival vessels. Seidel testing show's diffuse transconjunctival drainage. Intraocular pressure is $12 \mathrm{mmHg}$. success rate of filtering surgery for glaucoma. Treatment is given on a daily basis for one week and on alternative days for two weeks and this is disadvantageous for the patient, either requiring prolonged hospital stay or multiple hospital visits in the first two weeks after surgery.

Complications may be divided into early and late. Early complications include corneal erosions, early bleb leakage and bleb rupture while late complications are related to the development of cystic blebs.

5-Fluorouracil is given as subconjunctival injections as topical therapy is known to cause corneal epithelial erosions. Inevitably the drug will leak through the conjunctival needle track and into the tear film and corneal problems are common even with this relatively low dose regime. Serious corneal complications have been reported in eyes with preexisting corneal disease $\mathrm{10.11.19}^{10}$ and although none occurred in this series corneal complications were common and caused much discomfort.

Corneal endothelial cell counts have been reported to be unaffected by treatment with subconjunctival 5-fluorouracil ${ }^{12}$ and experimental work confirms that only high aqueous concentrations, much higher than encountered after subconjunctival injections of $5 \mathrm{mg}$, may be toxic. ${ }^{20}$

Early bleb leakage occurs more commonly in eyes treated with postoperative 5-fluorouracil compared to control eyes. ${ }^{11.12}$ This may be due to inhibition of fibroblast activity and scarring along the conjunctival incision line. Only one eye required surgical intervention to overcome a flat anterior chamber.

Several cases of sudden breakdown of conjunctiva overlying the bleb occurring in the first few weeks after surgery, and unrelated to the conjunctival incision, have been reported in association with the use of 5-fluorouracil and the condition has been termed 'bleb rupture'. ${ }^{10.12}$, 5-fluorouracil is known to be toxic to conjunctival as well as corneal epithelium and this may contribute to thinning and 'rupture' of conjunctiva overlying a drainage bleb. This is potentially very serious. Shallowing of the anterior chamber may cause corneal decompensation, formation of peripheral anterior synechiae and hasten the development of cataract. In additon the sudden loss of 
pressure increases the risk of choroidal detachment and delayed suprachoroidal haemorrhage.

Late complications of 5-fluorouracil encountered were related to the development of thin cystic blebs. Bleb morphology following 5-fluorouracil injection has not been well reported. In one small controlled trial of the use of 5-fluorouracil, 10 of 14 cases developed cystic blebs compared to one of 12 controls. Ten of the controls suffered fibrosis of the bleb and failure to control intraocular pressure and the failure of drainage may explain the low incidence of cystic blebs in the control group 13.

Cystic bleb formation is not uncommon after successful trabeculectomy. Our control group is unsatisfactory as they were reviewed retrospectively when it was suspected that a higher incidence of thin cystic blebs was occurring. Our control group patients were still attending the glaucoma unit and a note of the appearance of the bleb was made in all cases. Cystic blebs were noted in $20 \%$ of cases in the control group compared to $40 \%$ in the 5-fluorouracil treated group. Aqueous humour has been shown to have an inhibitory effect on fibroblast proliferation ${ }^{21}$ and it has been suggested that this inhibits collagen production in the subconjunctival space ${ }^{22}$ favouring the formation of cystic blebs. 5-fluorouracil is known to inhibit fibroblast formation and may be potentiating the effect of aqueous on the tissues of the subconjunctival space.

Thin cystic blebs are associated with transconjunctival drainage of aqueous humour into the tear film. Several cases treated with 5-fluorouracil showed scarring around the conjunctival bleb with a thin cystic central area and good intraocular pressure control because of transconjunctival drainage. (Fig 2) Transconjunctival drainage through thin cystic blebs was associated with intraocular pressures of less than $9 \mathrm{mmHg}$ in 10 eyes $(20 \%)$ in the 5-fluorouracial group compared to three eyes $(7 \%)$ in the control group. Most reports of the use 5-fluorouracil report mean intraocular pressure following surgery rather than the range of pressures encountered, however, Taniguchi reported 11 of 33 eyes $(33 \%)$ with intraocular pressures of $10 \mathrm{mmHg}$ or less with a mean follow up of 13 months after 5-fluorouracil treatment.

Five eyes $(15 \%)$ in the latter series and six eyes $(12 \%)$ in the series reported here had intraocular pressures of less than $6 \mathrm{mmHg}$ at which level there is a risk of chronic disc and macular oedema and fluctuating visual acuity. One eye lost one line of visual acuity, from $6 / 18$ to $6 / 24$ due to chronic macular oedema. No eye suffered total loss of the visual field as a result of surgery although this is a well recognised complication of trabeculectomy in eyes with advanced field loss. Two eyes with early failure due to fibrous scarring of the conjunctival bleb, subsequently lost further field of vision and central visual acuity, and required tube implant surgery.

Exuberant cystic blebs may be complicated by adjacent corneal dellen formation which may be uncomfortable as well as making contact lens wear difficult.

Full thickness drainage procedures are frequently complicated by cystic bleb formation and have an increased incidence of bleb infections and late cases of endophthalmitis than is the case with trabeculectomy with a guarded sclerostomy. Thin cystic blebs complication 5-fluorouracil treatment may be prone to similar complications.

\section{Conclusions}

The use of 5-fluorouracil to improve the chances of successful drainage surgery is well established. Complications of treatment include corneal erosions, early bleb leakage, and the development of thin cystic blebs. There is a high incidence of complications associated with the use of 5-fluorouracil and it should be reserved for complicated cases undergoing trabeculectomy where the risk of failure due to fibrosis and scarring of the conjunctival bleb is known to be high.

Further studies are required to assess the long-term complications of 5-fluorouracil, in particular those associated with the development of thin avascular cystic conjunctival blebs.

\footnotetext{
References

${ }^{1}$ Mills KB: Trabeculectomy: a retrospective longterm follow-up of 444 cases. Br J Ophthalmol 1981, 65: 790-5.

${ }^{2}$ Migdal C and Hitchings RA: Results of primary tra-
} 
beculectomy, a five year follow-up. Proceedings of the 14th International Congress of Ophthalmology, Singapore. 1990. (In press).

${ }^{3}$ Watson PG and Grierson I: The place of trabeculectomy in the treatment of glaucoma. Ophthalmology 1981, 88: 175-96.

${ }^{4}$ Beauchamp GR and Parks MM: Filtering surgery in children; barristers to success. Ophthalmology 1979, 86: 170-80.

${ }^{5}$ Gressel MG, Heuer DK, Parrish RK: Trabeculectomy in young patients. Ophthalmology 1984, 91: 378-83.

${ }^{6}$ Kolker AE and Hetherington J: Becker-Shaffer's Diagnosis and Therapy of the Glaucoma. St Louis, CV Mosby, 1983, pp 453-454.

${ }^{7}$ Miller RD and Barber JC: Trabeculectomy in black patients. Ophthalmic Surg 1981, 12: 46-50.

${ }^{8}$ Hoskins HD, Hetherington J, Shaffer RN: Surgical management of the inflammatory glaucomas. Perspect Ophthalmol 1977, 1: 173-81.

${ }^{9}$ Heuer DK, Parrish RK, Gressel MG, Hodapp E, Desjardins DC, Skuta GL, Palmberg PF, Nevarez JA, Rockwood EJ: 5-fluorouracil and glaucoma filtering surgery. Intermediate follow-up of a pilot study. Ophthalmology 1986, 93: 1537-46.

${ }^{10}$ Rockwood EJ, Parrish RK, Heuer DK, Skuta GL, Hodapp E, Palmberg PF, Gressel MG, Feuer W: Glaucoma filtering surgery with 5-fluorouracil. Ophthalmology 1987, 94: 1071-8.

${ }^{11}$ Taniguchi T, Kitazawa Y, Shimizu U: Long-term results of 5-fluorouracil trabeculectomy for primary open-angle glaucoma. Inter Ophthalmol 1989, 13: 145-9.

${ }^{12}$ The fluorouracil study group. Fluorouracil filtering surgery study one-year follow-up. Am J Ophthalmol 1989, 108: 625-35.

${ }^{1.3}$ Ruderman JM, Welch DB, Smith MF, Shoch DE: A randomised study of 5-fluorouracil and filtration surgery. Am J Ophthalmol 1987, 104: 218-24.

${ }^{14}$ Rabowsky JH and Ruderman JM: Low-dose 5-fluorouracil and glaucoma filtering surgery. Ophthalmic Surg 1989, 20: 347-9.

${ }^{15}$ Krug JH and Melamed S: Adjunctive use of delayed and adjustable low-dose 5-fluorouracil in refractory glaucoma. Am J Ophthalmol 1990, 109: 412-18.

${ }^{16}$ Weinreb RN: Adjusting the dose of 5-fluorouracil after filtration surgery to minimise side effects. Ophthalmology 1987, 94: 564-70.

${ }^{17}$ Cairns JE: Trabeculectomy: Preliminary report of a new method. Am J Ophthalmol 1968, 5: 673-9.

${ }^{18}$ Longstaff S, Wormald RPL, Masover A, Hitchings RA. Glaucoma Triple Procedure, results and efficacy of intraocular pressure control and visual outcome. Ophthalmic Surg (in press) 1990.

${ }^{19}$ Knapp A, Heuer DK, Stern G, Driebe WT: Serious corneal complications of glaucoma filtering surgery with postoperative 5-fluorouracil. Am J Ophthalmol 1987, 103: 183-7.

${ }^{20}$ Mannis MJ, Sweet EH, Lewis RA. The effect of fluorouracil on the corneal endothelium Arch Ophthalmol 1988, 106: 816-17.

${ }^{21}$ Kornbleuth W and Tenenbaum E: The inhibitory effect of aqueous humour on the growth of cells in tissue cultures. Am J Ophthalmol 1956, 42: 70-4.

${ }^{22}$ Teng CC, Chi HH, Katzin HM. Histology and mechanism of filtering operations. Am J Ophthamol 1959, 47: 16-34. 